\title{
On non-uniqueness of percolation on nonamenable Cayley graphs*
}

\author{
Igor PAK $^{\text {a }}$, Tatiana SMIRNOVA-NAGNIBEDA ${ }^{\text {b }}$ \\ ${ }^{\text {a }}$ Department of Mathematics, Yale University, New Haven, CT 06520, USA \\ E-mail: paki@math.yale.edu \\ ${ }^{\text {b }}$ Department of Mathematics, Royal Institute of Technology, 10044 Stockholm, Sweden \\ E-mail: tatiana@math.kth.se
}

(Reçu et accepté le 31 janvier 2000)

\begin{abstract}
The aim of this Note is to prove a weak version of the conjecture of Benjamini and Schramm about phase of non-uniqueness for the Bernoulli bond percolation on nonamenable transitive graphs. We show that every nonamenable finitely generated group has a finite system of generators such that the Bernoulli bond percolation on the corresponding Cayley graph has a nonempty non-uniqueness phase. Together with previously known results, this gives a characterization of amenability of finitely generated groups in terms of uniqueness of percolation. ( 2000 Académie des sciences/Éditions scientifiques et médicales Elsevier SAS
\end{abstract}

\section{Sur la non-unicité de percolation dans des graphes de Cayley non moyennables}

Résumé. Le but de la présente Note est de démontrer une version faible de la conjecture de Benjamini et Schramm sur la non-unicité de la percolation dans des graphes transitifs non moyennables. Nous montrons que chaque groupe non moyennable de type fini possède un système fini symétrique de générateurs avec la propriété que la phase de non-unicité est non vide pour la percolation de Bernoulli sur les arêtes du graphe de Cayley correspondant. (C) 2000 Académie des sciences/Éditions scientifiques et médicales Elsevier SAS

\section{Version française abrégée}

Dans cette Note, nous nous intéressons au problème d'unicité du cluster infini pour la percolation de Bernoulli sur les arêtes d'un graphe de Cayley non moyennable.

Soit $G$ un groupe infini de type fini, et soit $S$ un système fini symétrique de générateurs de $G$, de cardinalité $d$. Le graphe de Cayley de $G$ associé à $S$ est noté $\Gamma$.

La conductance $\varphi=\varphi(G, S)$ de $\Gamma$ est définie $\operatorname{par} \varphi=\inf _{X \subset G}\left|\partial_{E} X\right| / d \cdot|X|$, où $\partial_{E} X=\{(x, y) \mid x \in X$, $y \in G \backslash X\}$, et l'infimum est pris sur tous les sous-ensembles finis $X$.

\footnotetext{
Note présentée par Étienne GHYS.
} 


\section{Pak, T. Smirnova-Nagnibeda}

Le rayon spectral $\rho=\rho(G, S)$ est défini par $\rho=\limsup _{n \rightarrow \infty}\left(p^{(n)}(x, y)\right)^{1 / n}$, où $p^{(n)}(x, y)$ est la probabilité que la marche aléatoire simple, débutant au point $x \in G$, se trouve au point $y \in G$ après $n$ pas. Il est bien connu que le rayon spectral ne dépend pas de $x, y \in G$.

Les conditions suivantes sont équivalentes : (i) $G$ est non moyennable; (ii) $\rho(G, S)<1$ pour tout $S$ fini symétrique (voir [9]); (iii) $\varphi(G, S)>0$ pour tout $S$ fini symétrique (voir e.g. [5]).

Soit $0 \leqslant p \leqslant 1$. Chaque arête $(g, g s)$ de $\Gamma$ est indépendemment ouverte avec probabilité $p$ ou fermée avec probabilité $1-p$. Un cluster est une composante connexe du sous-graphe de $\Gamma$ formé d'arêtes ouvertes. Nous dirons que la percolation a lieu si l'identité de $G$ appartient à un cluster infini avec une probabilité $\theta(p)$ positive. Nous considérons également la probabilité $\zeta(p)$ que le cluster infini est unique. Les valeurs critiques de ces probabilités

$$
p_{\mathrm{c}}(G, S)=\sup \{p \mid \theta(p)=0\}, \quad p_{\mathrm{u}}(G, S)=\inf \{p \mid \zeta(p)=1\},
$$

présentent un intérêt particulier.

Il est démontré $[1,2,4]$ que si $G$ est moyennable, alors $p_{\mathrm{c}}(G, S)=p_{\mathrm{u}}(G, S)$ pour tout système fini symétrique de générateurs $S$. Benjamini et Schramm ont conjecturé dans [2] que la réciproque est vraie.

Conjecture 1 (Benjamini, Schramm). - Soit $G$ un groupe non moyennable, soit $S$ un système fini symétrique de générateurs de $G$. Alors $p_{\mathrm{c}}(G, S)<p_{\mathrm{u}}(G, S)$.

Dans cette Note, nous démontrons le résultat suivant.

Soit $S$ un système fini symétrique de générateurs de $G$. Pour tout entier $k \geqslant 1$, l'ensemble $S^{(k)}$ de cardinalité $d^{k}$, est constitué de tous les éléments $g \in G$ de norme inférieure ou égale à $k$ dans la métrique des mots induite par $S$, chacun des éléments étant pris autant de fois qu'il y a de manières différentes de l'écrire comme produit de générateurs de $S$.

THÉORÈmE 2. - Soit $G$ un groupe non moyennable, soit $S$ un système fini symétrique de générateurs de G. Alors $p_{\mathrm{c}}\left(G, S^{(k)}\right)<p_{\mathrm{u}}\left(G, S^{(k)}\right)$ pour tout $k>\log 2 / \log (1 / \rho(G, S))$.

Combiné au résultat cité ci-dessus sur les graphes moyennables, ce théorème implique une caractérisation des groupes moyennables en termes de percolation. Plus précisément : un groupe infini de type fini $G$ est moyennable si et seulement si $p_{\mathrm{c}}(G, S)=p_{\mathrm{u}}(G, S)$ pour tout système fini symétrique de générateurs $S$ de $G$.

La preuve du théorème est basée sur le lemme suivant.

LEMME 2. - Soit $G$ un groupe non moyennable. Alors $\lim _{k \rightarrow \infty} \rho\left(G, S^{(k)}\right)=0$ et $\lim _{k \rightarrow \infty} \varphi\left(G, S^{(k)}\right)=1$.

Nous utilisons aussi deux résultats de Benjamini et Schramm [2].

LEMME 3. - Si $\rho(G, S) \cdot p_{\mathrm{c}}(G, S) \cdot d<1$, alors $p_{\mathrm{c}}(G, S)<p_{\mathrm{u}}(G, S)$.

LEMME 4. - On a $p_{\mathrm{c}}(G, S) \leqslant 1 /(d \cdot \varphi(G, S)+1)$.

Nous présentons aussi deux autres conditions suffisantes pour qu'un graphe de Cayley ait une phase non vide de non-unicité de percolation.

\section{Introduction}

In the past few years there has been an explosion in the number of results dealing with percolation on transitive graphs. We refer to [6] for a general treatment of percolation (mostly on $\mathbb{Z}^{d}$ ), to [2,3] for other groups, and to [10] for percolation and other probabilistic processes on nonamenable groups. 
The problem of uniqueness of infinite cluster for percolation on $\mathbb{Z}^{d}$ was open for a long time until an elaborate solution was found in [1]. In [7], Grimmett and Newman discovered that for a product of $\mathbb{Z}$ and a regular tree of sufficiently large degree, the Bernoulli percolation has radically different behavior, namely, there are infinitely many infinite clusters for $p$ close to the critical value $p_{\mathrm{c}}$, but only one "giant" infinite cluster for $p$ close to 1 .

In a pioneer paper [2], Benjamini and Schramm introduced and provided a unified setting for a host of problems related to percolation on transitive graphs (see also [3] for the survey of recent progress and relevant references). They observed that the work of Burton and Keane [4] can be easily generalized to show the uniqueness of infinite cluster for percolation on all amenable transitive graphs. Benjamini and Schramm conjectured in [2] that for all nonamenable transitive graphs the Bernoulli percolation has a nonempty nonuniqueness phase, and proved that this is true for all nonamenable transitive planar graphs.

In this paper, we present a proof of the following statement which, together with the result on amenable Cayley graphs mentioned above, can be regarded as a percolation criterion of group amenability: every nonamenable group has a finite set of generators such that the Bernoulli bond percolation on the corresponding Cayley graph has a nonempty non-uniqueness phase.

The proof uses results from [2] and is based on the fact that the spectral radius of a Cayley graph of a nonamenable group is not bounded away from 0 (or equivalently, the conductance is not bounded away from 1) uniformly over all finite symmetric generating sets.

More careful analysis of behavior of random walks on Cayley graphs allows to obtain new information on the dependence of the isoperimetric constant on the generating set. One result is stated here, but more details and proofs will be given in the forthcoming paper [12].

\section{Definitions and main results}

Let $G$ be an infinite, finitely generated group, and let $S$ be a finite symmetric multiset of generators of cardinality $d$. (This means that we allow $S$ to contain several copies of the same generator. From now on we will use the word "set" for simplicity, though all our sets of generators will indeed be multisets.) Denote by $\Gamma=\Gamma(G, S)$ the Cayley graph of $G$ with respect to $S$.

Define the conductance $\varphi=\varphi(G, S)$ as

$$
\varphi=\inf _{X \subset G} \frac{\left|\partial_{E} X\right|}{d \cdot|X|},
$$

where $\partial_{E} X=\{(x, y) \mid x \in X, y \in G \backslash X\}$ and the infimum is taken over all finite sets $X$.

The spectral radius $\rho=\rho(G, S)$ is $\rho=\limsup _{n \rightarrow \infty}\left(p^{(n)}(x, y)\right)^{1 / n}$, where $p^{(n)}(x, y)$ is the probability that the simple random walk on $\Gamma$ starting at $x \in G$ reaches $y \in G$ after $n$ steps. It is independent of $x, y \in G$.

The following conditions are equivalent for a finitely generated group $G$ :

(i) $G$ is nonamenable;

(ii) $\rho(G, S)<1$ for any finite symmetric generating set $S$ (see [9]);

(iii) $\varphi(G, S)>0$ for any finite symmetric generating set $S$ (see, e.g., [5]).

LEMMA 1 (Mohar [11]). - The constants $\rho, \varphi$, and $d$ are related by the following inequalities:

$$
\begin{aligned}
& \varphi \geqslant \frac{d(1-\rho)}{d-1} \quad\left(\text { equivalently } \rho \geqslant 1-\frac{\varphi(d-1)}{d}\right), \\
& \left.\varphi \leqslant \sqrt{1-\rho^{2}} \quad \text { (equivalently } \rho \leqslant \sqrt{1-\varphi^{2}}\right) .
\end{aligned}
$$

Let $0 \leqslant p \leqslant 1$. The Bernoulli bond percolation on $\Gamma$ is defined as follows. An edge $(g, g s)$ is open with probability $p$ and closed with probability $1-p$. Connected components of the subgraph spanned by 


\section{Pak, T. Smirnova-Nagnibeda}

the open edges are called open clusters. Denote by $\theta(p)$ the probability that the identity element $i d \in G$ belongs to an infinite open cluster. Denote by $\zeta(p)$ the probability that there exists exactly one infinite open cluster. Consider the corresponding critical values:

$$
p_{\mathrm{c}}(G, S)=\sup \{p \mid \theta(p)=0\}, \quad p_{\mathrm{u}}(G, S)=\inf \{p \mid \zeta(p)=1\} .
$$

It is easy to see that $\theta(p)$ is nondecreasing (see, e.g., [6] p. 13). It is known that for Cayley graphs $\zeta(p)=1$ if $p>p_{\mathrm{u}}$ (see, e.g., [8]). Thus we obtain three intervals for the percolation behavior: $\left(0, p_{\mathrm{c}}\right),\left(p_{\mathrm{c}}, p_{\mathrm{u}}\right),\left(p_{\mathrm{u}}, 1\right)$, where the percolation a.s. has $0, \infty, 1$ infinite open clusters respectively (see [2]). The following conjecture is due to Benjamini and Schramm (see [2,3]):

Conjecture 1 (Benjamini, Schramm). - Let $G$ be a nonamenable group, and let $S$ be a finite symmetric set of generators in $G$. Then $p_{\mathrm{c}}(G, S)<p_{\mathrm{u}}(G, S)$.

We now state our main result.

THEOREM 1. - Let $G$ be a nonamenable group. Then there exists a finite symmetric set of generators $S$ in $G$ such that $p_{\mathrm{c}}(G, S)<p_{\mathrm{u}}(G, S)$.

Let $S \subset G$ be any finite symmetric generating set. For any integer $k \geqslant 1$, define $S^{(k)}$ to be the multiset of cardinality $d^{k}$ which consists of all elements $g \in G$ of length less or equal to $k$ in the word metric given by $S$, each taken with multiplicity equal to the number of possible ways to write $g$ as a product $g=s_{1} \cdots s_{k}$ with $s_{1}, \ldots, s_{k} \in S$. Obviously, any $S^{(k)}$ generates $G$.

THEOREM 2. - Let $G$ be a nonamenable group, let $S$ be a finite symmetric set of generators in $G$. Then $p_{\mathrm{c}}\left(G, S^{(k)}\right)<p_{\mathrm{u}}\left(G, S^{(k)}\right)$ for all $k>\log 2 / \log (1 / \rho(G, S))$.

The proof of Theorem 2, which of course implies Theorem 1, makes use of the following fact.

Lemma 2. - Let $G$ be a nonamenable group. Then $\lim _{k \rightarrow \infty} \rho\left(G, S^{(k)}\right)=0$ and $\lim _{k \rightarrow \infty} \varphi\left(G, S^{(k)}\right)=1$.

More elaborate arguments lead to a quantitative version of Lemma 2, as follows.

THEOREM 3. - Let $G$ be a nonamenable group, let $S$ be a finite symmetric set of generators in $G$. Then, for any $\varepsilon>0$, one has $\varphi\left(G, S^{(k)}\right)>1-\varepsilon$, given $k>C / \varphi^{2} \log (1 / \varepsilon)$ where $C$ is a universal constant.

This leads to the following version of Theorem 2 .

THEOREM 4. - Let $G$ be a nonamenable group, let $S$ be a finite symmetric set of generators in $G$. Then $p_{\mathrm{c}}\left(G, S^{(k)}\right)<p_{\mathrm{u}}\left(G, S^{(k)}\right)$, given $k>C / \varphi(G, S)^{2}$, where $C$ is a universal constant.

\section{Uniqueness of percolation}

We start by proving Lemma 2.

Proof of Lemma 2. - Denote by $M(G, S)$ the Markov operator of averaging over generators on the Cayley graph $\Gamma(G, S)$. The spectral radius of the graph is then equal to the norm of $M$ as of self-adjoint operator on the Hilbert space $\ell^{2}(G)$. As the group $G$ is nonamenable, the spectral radius of $\Gamma$ is strictly less than 1 . The norm of the $k$-th power of $M(G, S)$ is exactly the Markov operator on the Cayley graph of $G$ with respect to the generating set $S^{(k)}$. Therefore we have

$$
\rho\left(G, S^{(k)}\right)=\left\|M\left(G, S^{(k)}\right)\right\|_{\ell^{2}(G)}=\left\|M(G, S)^{k}\right\|_{\ell^{2}(G)} \leqslant\|M(G, S)\|_{\ell^{2}(G)}^{k}=\rho(G, S)^{k} \underset{k \rightarrow \infty}{\longrightarrow} 0 .
$$


In order to prove the second part of Lemma, we apply the inequality (1) to $\varphi\left(G, S^{(k)}\right)$ and get

$$
\varphi\left(G, S^{(k)}\right) \geqslant \frac{d^{k}}{d^{k}-1}\left(1-\rho\left(G, S^{(k)}\right)\right) \underset{k \rightarrow \infty}{\longrightarrow} 1
$$

Besides Lemma 2, the following results will be used in the proof of Theorem 2.

LEMMA 3 (Benjamini, Schramm). - If

$$
\rho(G, S) p_{\mathrm{c}}(G, S) d<1,
$$

then $p_{\mathrm{c}}(G, S)<p_{\mathrm{u}}(G, S)$.

LEMMA 4 (Benjamini, Schramm). - We have

$$
p_{\mathrm{c}}(G, S) \leqslant \frac{1}{d \cdot \varphi(G, S)+1} .
$$

Both results are proven in [2] for the Bernoulli site percolation. For the bond percolation, the first proof goes through verbatim, and the second can be adopted without difficulty.

Proof of Theorem 2. - We will show that $\rho\left(G, S^{(k)}\right) p_{\mathrm{c}}\left(G, S^{(k)}\right) d^{k} \underset{k \rightarrow \infty}{\longrightarrow} 0$. Theorem then follows from Lemma 3. Lemma 4, the inequality (1), and Lemma 2 imply:

$$
\begin{aligned}
\rho\left(G, S^{(k)}\right) p_{\mathrm{c}}\left(G, S^{(k)}\right) d^{k} & <\rho\left(G, S^{(k)}\right) \frac{1}{d^{k} \varphi\left(G, S^{(k)}\right)} d^{k} \\
& \leqslant \rho\left(G, S^{(k)}\right) \frac{d^{k}-1}{d^{2 k}\left(1-\rho\left(G, S^{(k)}\right)\right)+d^{k}-1} d^{k} \\
& <\rho\left(G, S^{(k)}\right) \frac{1}{\frac{d^{k}}{d^{k}-1}\left(1-\rho\left(G, S^{(k)}\right)\right)+\frac{1}{d^{k}-1}-\frac{1}{d^{k}}} \underset{k \rightarrow \infty}{\longrightarrow} 0 .
\end{aligned}
$$

We see that for large values of $k$, the product $\rho\left(G, S^{(k)}\right) p_{\mathrm{c}}\left(G, S^{(k)}\right) d^{k}$ is strictly less than 1 . In particular, the inequality (2) is satisfied for every $\Gamma\left(G, S^{(k)}\right)$ with $k>\log 2 / \log (1 / \rho(G, S))$.

Proof of Theorem 3 will be given in [12]. We present below two estimates of independent interest which can be used in the proof of Theorem 4. The first of them was obtained independently by R. Schonmann.

Proposition 1. - Let $G$ be a finitely generated group, $S$ a finite symmetric generating set.

(i) Assume that $\varphi(G, S)>1 / \sqrt{2}$. Then $p_{\mathrm{c}}(G, S)<p_{\mathrm{u}}(G, S)$.

(ii) Assume that $\rho<\left(d^{2}+d-1\right) /\left(2 d^{2}-d\right)$. Then $p_{\mathrm{c}}(G, S)<p_{\mathrm{u}}(G, S)$.

Proof. - By inequality (1), $\rho(G, S) \leqslant \sqrt{1-\varphi(G, S)^{2}}<1 / \sqrt{2}$. By Lemma $4, p_{\mathrm{c}}(G, S) \leqslant 1 /(d$. $\varphi(G, S)+1)<\sqrt{2} /(d+\sqrt{2})$. We conclude that $\rho(G, S) \cdot p_{\mathrm{c}}(G, S) \cdot d<d /(d+\sqrt{2})<1$, and by Lemma 3 the proof is complete. Similar proof works for the second statement. Note that the second assumption is satisfied if $\rho<1 / 2$, independently of $d$.

Remark. - The set $S^{(k)}$ can be viewed as the ball $B_{k}$ of radius $k$ in the graph $\Gamma$ where each element has been assigned a weight equal to the number of paths of length $k$ in $\Gamma$ connecting $i d$ and this element. It is natural to ask whether in Lemma 2 one can put all weights equal to 1 and consider the balls $\left\{B_{k}\right\}_{k \geqslant 1}$ (or some subsets of them) instead of $\left\{S^{(k)}\right\}_{k \geqslant 1}$. The answer appears to be positive for many groups. 


\section{Pak, T. Smirnova-Nagnibeda}

Example 1.-Groups with free subgroups. ${ }^{1}$ If $G$ contains a free subgroup then it contains a free subgroup $G_{m}$ on any number $m \in \mathbb{N}$ of generators. Denote by $\mathcal{A}_{m} \subset G$ a set of elements of $G$ which freely generate $G_{m}$. Consider symmetric generating sets $\widetilde{\mathcal{A}}_{m}=S \cup \mathcal{A}_{m} \cup \mathcal{A}_{m}^{-1}$ in $G$. Every $\Gamma\left(G_{m}, \mathcal{A}_{m}\right)$ is a spanning tree in the Cayley graph $\Gamma\left(G, \widetilde{\mathcal{A}}_{m}\right)$. The graph is homogeneous of degree $2 m+2+|S|$ and the spanning tree is homogeneous of degree $2 m+2$. Therefore $\rho\left(G, \widetilde{\mathcal{A}}_{m}\right)-\rho\left(\mathbb{F}_{m}\right) \underset{m \rightarrow \infty}{\longrightarrow} 0$. As $\rho\left(\mathbb{F}_{m}\right)=\sqrt{2 m-1} / m$, we get $\rho\left(G, \widetilde{\mathcal{A}}_{m}\right) \underset{m \rightarrow \infty}{\longrightarrow} 0$.

Example 2. - Groups with rapid decay property. A group is said to have (RD)-property if the Haagerup inequality $\|\Phi\|^{2} \leqslant C \sum_{g \in G}|\phi(g)|^{2}\left(1+|g|^{2}\right)^{2 \delta}$ is satisfied for every operator $\Phi$ of convolution with a function $\phi \in \ell^{2}(G)$, with constants $C=C(G), \delta=\delta(G)$. Recall that the class of groups with (RD)property is closed under direct product and contains all word hyperbolic groups. It also includes some interesting examples of groups acting on buildings (see [13] $)^{2}$.

Suppose $G$ has (RD)-property. For $k \geqslant 1$, let $B_{k}, \Lambda_{k}$ denote respectively the ball and the sphere of radius $k$ in the Cayley graph $\Gamma=\Gamma(G, S)$ for some finite symmetric $S$. The (RD)-property implies

$$
\rho\left(G, B_{k}\right)=\left\|\frac{1}{\left|B_{k}\right|} \sum_{g \in B_{k}} g\right\| \leqslant \frac{C}{\left|B_{k}\right|} \sqrt{\sum_{i=1}^{k}(1+i)^{2 \delta}\left|\Lambda_{i}\right|} \leqslant \frac{C}{\left|B_{k}\right|} \sum_{i=1}^{k}(1+i)^{\delta} \sqrt{\left|\Lambda_{i}\right|} \underset{k \rightarrow \infty}{\longrightarrow} 0 .
$$

Acknowledgement. We would like to thank Itai Benjamini and Oded Schramm for the introduction to the subject and for the valuable remarks on the preliminary version of this paper. The comments of Pierre de la Harpe, Russel Lyons, and Yuval Peres were also much appreciated. The first author is grateful to László Babai, Gene Cooperman, László Lovász and Alistair Sinclair for very helpful conversations. The second author would like to acknowledge a valuable discussion with Andrzej Żuk who showed us a short proof of Lemma 2 presented above.

Both authors were supported by the Swedish government grant for collaboration between Yale University and KTH Stockholm. We are also grateful to the organizers of the Workshop on group theory and geometry in the University of Warwick where part of this work was completed.

\footnotetext{
* The first author was supported by NSF Postdoctoral Research Fellowship. The second author was supported by a postdoctoral fellowship from G. Gustafsson Foundation.

1 This argument was first shown to us by Itai Benjamini and Oded Schramm.

2 We are grateful to Alain Valette for a discussion on groups with rapid decay property.
}

\section{References}

[1] Aizenman M., Kesten H., Newman C.M., Uniqueness of the infinite cluster and continuity of connectivity functions for short and long range percolation, Commun. Math. Phys. 111 (1987) 505-531.

[2] Benjamini I., Schramm O., Percolation beyond $\mathbb{Z}^{d}$, many questions and a few answers, Electron. Commun. Probab. 1 (1996) 71-82.

[3] Benjamini I., Schramm O., Recent progress on percolation beyond $\mathbb{Z}^{d}$, Preprint, 1999.

[4] Burton R.M., Keane M., Density and uniqueness in percolation, Commun. Math. Phys. 121 (1989) 501-505.

[5] Dodziuk J., Difference equations, isoperimetric inequality and transience of certain random walks, Trans. Amer. Math. Soc. 284 (1984) 787-794.

[6] Grimmett G., Percolation, Springer-Verlag, New York, 1989.

[7] Grimmett G., Newman C.M., Percolation in $\infty+1$ dimensions, in: Disorder in Physical Systems, Clarendon Press, Oxford, UK, 1990, pp. 219-240.

[8] Häggström O., Peres Y., Monotonicity of uniqueness for percolation on Cayley graphs: All infinite clusters are born simultaneously, Probab. Th. Rel. Fields 113 (1999) 273-285.

[9] Kesten H., Full Banach mean values on countable groups, Math. Scand. 7 (1959) 146-156.

[10] Lyons R., Phase transitions on nonamenable graphs, Preprint, 1999.

[11] Mohar B., Isoperimetric inequalities, growth, and the spectrum of graphs, Lin. Alg. Appl. 103 (1988) $119-131$.

[12] Pak I., Smirnova-Nagnibeda T., Isoperimetric behavior of Cayley graphs, (in preparation).

[13] Ramagge J., Robertson G., Steger T., A Haagerup inequality for $\widetilde{A}_{1} \times \widetilde{A}_{1}$ and $\widetilde{A}_{2}$ buildings, GAFA 8 (1998) 702-731. 